\title{
Intelligent Remote Control Device Based on Household Telephone Channel
}

\author{
Deng Peng \\ $\{$ dp12356@163.com $\}$ \\ College of Electronic and Information Engineering, Jingchu University of Technology, Jingmen \\ 448000, China
}

\begin{abstract}
This paper designs a kind of intelligent remote control device of household telephone channel decoding chip MT8870 based on the use of multi frequency signal decoding telephone sent into SCM system after processing, give the operator prompts by controlling ISD4004 chip voice recording playback speech, realize remote control of household appliances. After the actual test: the device is economic, safe and reliable, consistent with the appliance control development direction, has a certain practical value.
\end{abstract}

Keywords: Remote control; Household appliances; Dual tone and multi-frequency; Chip microcomputer; DTMF decode

\section{Introduction}

With the Internet network to the ordinary family life continues to expand, consumer electronics, computers, telecommunications integration is becoming increasingly apparent, intelligent information appliances products began to enter the community and family. Household appliances telephone remote control is a kind of remote control technology based on public telephone network system, compared to the telephone remote control and conventional control method has certain advantages, it does not need occupy the radio frequency resources, also need special wiring, reduce the electromagnetic pollution of the people's living space and the timeliness and reliability of system control is also very good [1]. At the same time, due to the local telephone line network, can make full use of existing telephone network, so the remote control distance can be across provinces and cities, and even across the country. The device with the family of fixed telephone network to transmit control command signal, the command was analyzed by single chip microcomputer system, the relay control of household appliances, without having to do less change for the fixed telephone or home appliances and convenient installation. 


\section{Overall Design Scheme}

The device to $51 \mathrm{SCM}$ as control core, using ordinary telephone sends the remote control command signal, without the need to install specialized remote launch device, with DTMF signal as the media carrier remote control command, with telephone remote appliance remote control switch function is realized by using a dual tone multi frequency receiver MT8870. Using the telephone network to transmit the remote control command or other digital information, the receiving device is mounted in a telephone subscriber line terminal, you can receive any place (as long as the phone can access the remote operation

Or other digital information, the receiving device is like an ordinary telephone set, the fax machine or other telephone user terminal equipment is used, so it is not subject to the limitations of distance. The device if detect ringing up to a certain number of times, if no answer, it will automatically pick and hang into the testing password, enter the correct selection control circuit, and then enter the on or off to control appliances, after the completion of the return. In order to ensure the safety of the equipment, the use of AT24C02 storage password and some initialization data and realize the automatic reset function of the watchdog $[2,3]$. In order to use the user operation interface is good, the voice chip prompt and the operation success feedback prompt function, the user operation is simple, reliable. The design of the telephone remote control schematic diagram shown in Fig. 1.

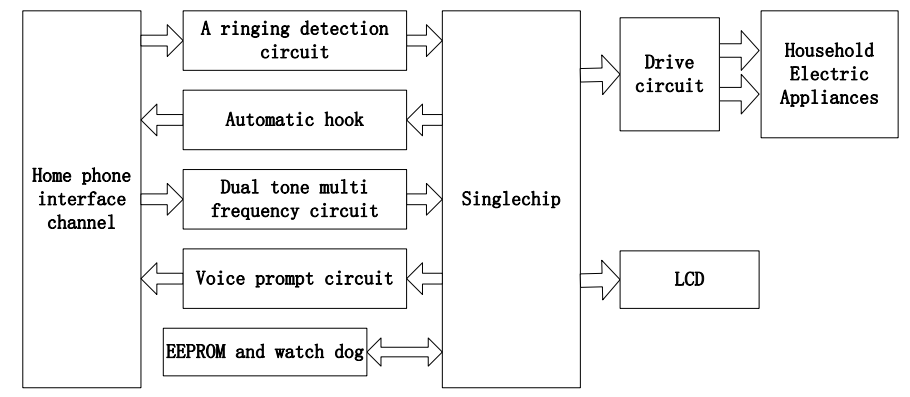

Fig. 1. Telephone remote control principle diagram

\section{System Hardware Circuit Design}

\subsection{Ringing Detection Circuit Design}

When there is no ringing signal, the line supply voltage is $+48 \mathrm{~V}$ (part of the switch is + $60 \mathrm{~V}$ ); when the user is called, program-controlled switches is sent to the called subscriber bell flow signal. Program-controlled switches to the user ringing voltage ringing is generally higher, the provisions of China's standard is $90 \mathrm{~V}+15 \mathrm{~V}, 25 \mathrm{~Hz}$ AC voltage as the bell current voltage, using $5 \mathrm{~S}$ intermittent, during which the $1 \mathrm{~s}$ to send, $4 \mathrm{~S}$, input and a photoelectric coupler [4]. The power supply voltage on the line designed by this system is $+/-48 \mathrm{~V}$. According to the characteristics of the ringing signal voltage is higher, can first through a bridge rectifier, the optocoupler output square wave pulse to monolithic integrated circuit processing. The ringing detection circuit principle diagram as shown in Fig. 2. 


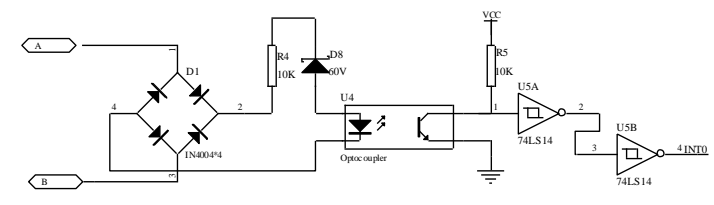

Fig. 2. A ringing detection circuit principle diagram

Have to be transformed into AC full when there is no ringing signal, line - 48V power supply voltage by a diode bridge rectifier, not enough to $60 \mathrm{~V}$ voltage regulator tube conduction, ringing signal output terminal voltage of $0 \mathrm{~V}$ when the ringing signal arrives, the line of $90 \mathrm{~V}$ ringing signal by the full bridge rectifier $90 \mathrm{~V}$ pulsating DC voltage, the peak to breakdown voltage value is $60 \mathrm{~V}$ voltage regulator tube, the resistance to the optocoupler provides voltage after Schmitt trigger two times, the output waveform shaping square wave signal can be directly output to the MCU interrupt input pin INT0, SCM according to the ringing signal after photoelectric conversion after the high-low detection without ringing.

\subsection{Simulation of Off Hook Circuit Design}

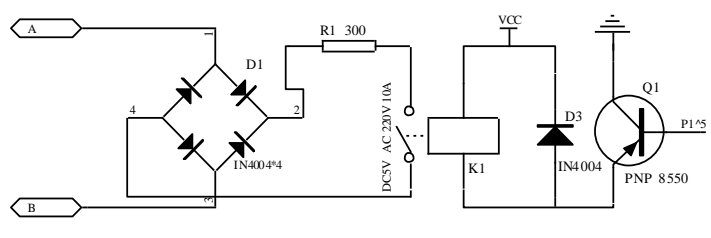

Fig. 3. Simulation off hook circuit diagram

When the MCU ringing pulse count value reaches the set value of the software, such as up to eight times, the SCM sends out off hook command. At this time relay K1, between a telephone subscriber line access to the $300 \mathrm{ohm}$ resistor. PBX user is detected between the line resistance decreases, that the called user is off hook and then he stopped ringing signal sending, communication between the two sides set up calls line, enter the normal call state. When the system completes the operation after the microcontroller sends hang orders, loss of power relay $\mathrm{K} 1$, disconnect the user line between $300 \mathrm{ohm}$ resistor, program-controlled switches detection to the changes that the user has hang up, and then cut off both sides of the communication line, the end of the call $[5,6]$. Simulation of off hook circuit principle diagram is shown in Fig. 3.

\subsection{MT8870 Dual Tone Multi Frequency Circuit Design}

Mitel company developed tone decoding chip MT8870, the chip can signal into high frequency band and low frequency band, and signal is sent to the digital decoder solution of key value, the signal in a binary way through Q1, Q2, Q3, Q4 four output pins. CPU read values when MT8870 solved a key value and the output to the outside, the STD pin will by low level up to a high level, after a period of lowered to a low level, we can use this feature 
detects this foot signal, Q1 Q4 horse will pin generated, although after solve a phone key value. Dual tone multi frequency circuit diagram is as shown in Figure 4, the DTMF signal sent by the user through the capacitor C5 and resistor R4 is coupled to the chip 2 feet (internal operational amplifier inverting input), inside the chip decoding. The circuit can receive the DTMF signal and is a complete DTMF receiver.

LNICST has integrated its copyright form into the paper submission system which means that you must agree with transferring the copyrights of your paper while uploading your camera ready version to the submission system. The author who uploads the paper should be the author who has the authority to agree with the terms of the copyright agreement on behalf of all the authors. After confirming the agreement the author will receive an e-mail with the filled document which is confirmed by both the author and ICST.

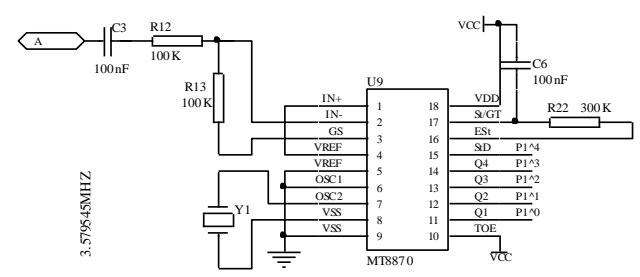

Fig. 4. Dual tone multi frequency circuit diagram

\subsection{Voice Prompt Circuit Design}

ISD4004 voice chip working circuit diagram shown in Fig. 5, ISD4004 work in the SPI serial interface. SPI is a synchronous serial data transmission protocol, protocol assumes that the micro controller SPI shift register in the SCLK falling along the action, so the ISD4004, clock stop rising edge latches MoSi pin data, on the falling edge of the data is sent to the miso pin.

The device is stored in the voice signal of the Chinese Communist Party: "Dear user, hello! You are welcome to use the phone intelligent control system, please enter the password "," whether to modify the password to modify the password, please press "1" key, please press "0" return "," wrong password, please input again, please enter the password, please enter the function keys, press' * 'key to end "," please enter the password to amend the four "and" password has been modified, please enter the function keys, press the' * 'key to the end "and" 1 home appliances have been opened, 1 home appliances have been closed, 2 home appliances have been opened, 2 home appliances have been closed, please input to control the time, press the' * 'key to the end "and" 3 home appliances have begun working time "," No. 4 appliance has begun working time "," No. 3 appliance has stopped working "," No. 4 appliance has stopped working ". 


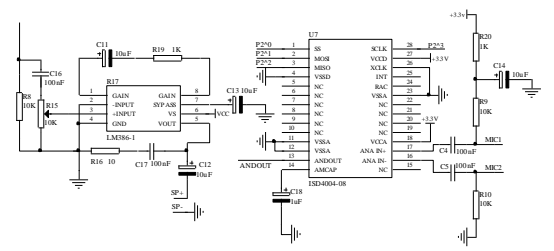

Fig. 5. ISD4004 voice chip working circuit diagram

\section{$4 \quad$ System Software Design}

After power on the system to enter the initial state, waiting for the user to call the phone control. When ringing to 5 times the system automatically off hook, and prompts the user to enter a password, all control operation only when the password is entered correctly, to take effect. After the password is entered correctly, the user will be prompted to change the password, can choose to modify or not modify, the system will automatically save the new password. After that the system prompt the user input function keys control home appliances, the home appliances and home appliance 4 operation timing, according to the "\#" can achieve hook [7]. The key operation time all over the system settings hang time, the system will be reset, the program will be executed from the beginning. The general flow chart of the program is shown in Fig. 6.

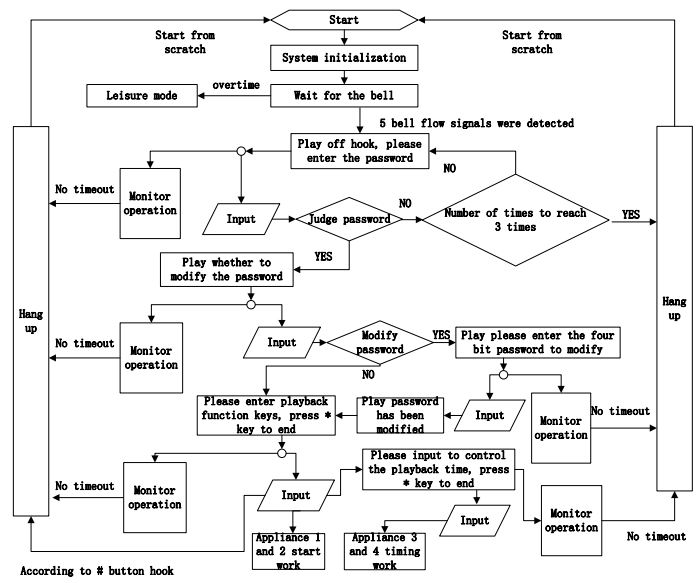

Fig. 6. The system program flow chart

\section{$5 \quad$ Concluding Remarks}

The design of single-chip microcomputer as the control core, users in the outdoor can use personal user terminals, such as fixed telephone, mobile phone, send DTMF signal, through the DTMF decoding chip MT8870 decoder, sent to single chip micro computer analysis and processing, to achieve a remote control of household appliances. In order to facilitate the 
operation of the user, the control system to add a voice prompt function. Voice chip under the control of the SCM play the desired speech, by controlling the voice recording chip ISD4004 playback speech signal gives the operator a prompt, the user can hear an individual user terminal equipment in the cue to discourse. This device can fully integrate the existing resources to play a greater function, to bring great convenience to people's lives.

\section{References}

[1] Zhang Meiqin, Gong Weiguo, Li Zhenghao, Zhou Liuyang. Design of the measurement and control technology of. remote control of household appliances and home security monitoring system based on smart phone, Vol. 26. No. 8, pp. 72 74, 86. (2007)

[2] Han Deqiang. The application and implementation of. Electronic technology design of embedded home controller system. No. 03, pp. 23 25, 28. (2008)

[3] $\mathrm{Wu}$. The public telephone network MCU control system design based on household appliances. Journal of Guangxi Normal University for Nationalities. No. 05, pp. 46 49. (2010)

[4] Yu Wenjun, Lexus Hao. Study on. Process automation instrumentation a IOT of intelligent Home Furnishing system, Vol. 32. No. 8, pp. 56 59. (2011)

[5] Guo Wentao. Research and design of. and computer measurement and control of. Intelligent home remote monitoring system. No. 09, pp. 2109 2112. (2011)

[6] Liu backbone, song Yue, He Zhihui, Wang Zhuohao. Intelligent remote home control wireless module and GSM realization of the measurement and control system of computer. No. 09, pp. 2133 2135, 2139. (2011)

[7] Deng Haitao, Wang Weifeng, Liu Qing. Remote control intelligent controller design for. Low voltage electric appliance based on. No. 14, pp. 16 18. (2007) 